\title{
Unveiling the Timescale of the R-T Transition in Human Hemoglobin
}

\author{
M. Cammarata ${ }^{1,2}$, M. Levantino ${ }^{3}$, M. Wulff $^{1}$ and A. Cupane ${ }^{3 *}$
}

${ }^{1}$ European Synchrotron

Radiation Facility,

Grenoble, France

${ }^{2}$ Centre for Molecular Movies,

University of Copenhagen,

Copenhagen, Denmark

${ }^{3}$ Department of Physical and

Astronomical Sciences,

University of Palermo,

Via Archirafi 36, I-90123

Palermo, Italy

Received 27 January 2010;

received in revised form

17 May 2010;

accepted 24 May 2010

Available online

1 June 2010

Edited by P. Wright
Time-resolved wide-angle X-ray scattering, a recently developed technique allowing to probe global structural changes of proteins in solution, was used to investigate the kinetics of R-T quaternary transition in human hemoglobin and to systematically compare it to that obtained with timeresolved optical spectroscopy under nearly identical experimental conditions. Our data reveal that the main structural rearrangement associated with the $\mathrm{R}-\mathrm{T}$ transition takes place $\sim 2 \mu \mathrm{s}$ after the photolysis of hemoglobin at room temperature and neutral $\mathrm{pH}$. This finding suggests that the 20- $\mu$ s step observed with time-resolved optical spectroscopy corresponds to a small and localized structural change.

(C) 2010 Elsevier Ltd. All rights reserved.

\section{Introduction}

Proteins can be considered as nanoscale molecular machines whose tertiary and quaternary structures are finely tailored to perform their biological task. In particular, it is well known that protein conformational changes are essential to optimize functional properties. ${ }^{1}$ A paradigmatic example is given by the allosteric control of enzyme function; the most widely studied case is probably that of the "honorary enzyme" hemoglobin $(\mathrm{Hb}) \cdot{ }^{2} \mathrm{Hb}$ is a tetrameric protein that is made of four subunits (two $\alpha$ chains and two $\beta$ chains) arranged as a dimer of two identical $\alpha \beta$ dimers; each subunit contains a heme group that is capable of reversibly binding exogenous ligands such as $\mathrm{O}_{2}$ or

${ }^{*}$ Corresponding author. E-mail address: cupane@fisica.unipa.it.

Present address: M. Cammarata, SLAC National Accelerator Laboratory, Menlo Park, CA, USA.

Abbreviations used: $\mathrm{Hb}$, hemoglobin; $\mathrm{CO}$, carbon monoxide; $\mathrm{HbCO}, \mathrm{CO}$ adduct of $\mathrm{Hb}$; TR-UVRR, time-resolved UV resonance Raman; TR-WAXS, time-resolved wide-angle $\mathrm{X}$-ray scattering; deoxyHb, deoxygenated $\mathrm{Hb}$; $\mathrm{SVD}$, singular value decomposition; metHb, methemoglobin. carbon monoxide (CO). The main physiological role of $\mathrm{Hb}$ is to act as oxygen carrier from the lungs to the tissues; in order to perform its task in an efficient way, $\mathrm{Hb}$ has to react with oxygen in a cooperative way. ${ }^{3}$ Thanks to the conceptual framework introduced by the Monod-Wyman-Changeux model ${ }^{4}$ and the seminal crystallographic work of Perutz, it is widely recognized that the "secret" of $\mathrm{Hb}$ cooperativity lies in the fact that this molecule is able to adopt at least two different quaternary structures in solution: a "relaxed" (R) structure stabilized by the presence of ligands, and a "tense" (T) structure that is stable when the protein is unligated. ${ }^{5}$ The ligated-to-unligated transition in $\mathrm{Hb}$ involves both conformational changes within the subunits (tertiary structure transition) and changes in the relative disposition of the subunits (quaternary structure transition). This last transition consists mainly in a $\sim 15^{\circ}$ rotation of one $\alpha \beta$ dimer with respect to the other in which interdimer interfaces are rearranged (whereas intradimer interfaces remain largely unchanged). As a result, the ligand affinity of the $\mathrm{R}$ quaternary conformation is greatly increased with respect to that of the T quaternary conformation. The $\mathrm{R}-\mathrm{T}$ transition is deemed to be mainly responsible for the cooperative behavior of $\mathrm{Hb}$ and is also often used as a paradigm of structure-mediated cooperativity in molecular biology. 
The pathway that connects the endpoint states of the allosteric transition in $\mathrm{Hb}$ (" $\mathrm{T}$ " and " $\mathrm{R}$ " states) has been extensively studied in the last decades, mainly using fast spectroscopic techniques such as laser flash photolysis of the $\mathrm{CO}$ adduct of $\mathrm{Hb}$ (HbCO). As early as 1976, Sawicki and Gibson, using time-resolved optical absorption spectroscopy, identified spectral relaxation consisting in a shift of the Soret band and used it to monitor the kinetics of $\mathrm{R} \rightarrow \mathrm{T}$ transition. ${ }^{6}$ Substantial improvements in the technique (both time resolution and data analysis) were made in the 1980s and allowed the identification of much finer details, including geminate recombination and further spectral relaxations. $^{7-10}$ The classically accepted picture emerging from those studies was that protein tertiary relaxation takes place in the submicrosecond timescale, while the quaternary R-T transition occurs at $\sim 20 \mu \mathrm{s}$ after photolysis at room temperature and neutral $\mathrm{pH}$. This picture was essentially confirmed by time-resolved resonance Raman in which a change in the heme resonance Raman spectrum with a time constant of 20-30 $\mu$ s, assigned to a tertiary structure relaxation close to the hemes, is thought to signal completion of the R-T quaternary structure change. ${ }^{11,12}$ More recently, timeresolved UV resonance Raman (TR-UVRR), timeresolved circular dichroism, and time-resolved magnetic circular dichroism have been used to complement optical absorption and resonance Raman data. ${ }^{13-15}$ TR-UVRR studies, besides identifying fast submicrosecond relaxations connected to the $\mathrm{E}$ and $\mathrm{F}$ helices clamshell rotation and to the breaking/reformation of interhelical hydrogen bonds, ${ }^{16}$ have indicated a stepwise quaternary transition in $\mathrm{Hb}$ in which a fast relaxation in $2 \mu \mathrm{s}$ is followed by a slower one in 20-50 $\mu$ s. TR-UVRR studies attributed the fast relaxation to the formation of quaternary H-bonds at the "hinge region" of the $\alpha_{1} \beta_{2}$ interface and suggested that dimer rotation is almost completed already at these short times; the slower 20- $\mu$ s relaxation was attributed to formation of contacts in the "switch region" of the $\alpha_{1} \beta_{2}$ interface and to tertiary relaxation at the hemes.

In contrast, time-resolved optical absorption spectroscopy, time-resolved circular dichroism, and time-resolved magnetic circular dichroism studies were interpreted in terms of a compound $\mathrm{R} \rightarrow \mathrm{T}$ transition beginning with a conformational relaxation localized at the $\alpha_{1} \beta_{2}$ interface, followed by the slower global relaxation of the protein structure to the equilibrium $\mathrm{T}$ form. ${ }^{14,15,17}$ In this picture, the relative rotation and translation of dimers, usually associated with the term "quaternary transition" in $\mathrm{Hb}$, were deemed to occur during the slow $\sim 20$ - $\mu$ s process. It appears therefore that a generally accepted picture of the time sequence of structural events involved in the transition from the $\mathrm{R}$ quaternary structure to the $\mathrm{T}$ quaternary structure of $\mathrm{Hb}$ is far from being reached. It should also be noted that while optical absorption spectroscopy is very sensitive to changes in the ligation state of $\mathrm{Hb}$, it is not a direct structural technique. Indeed, the structural relevance of a given observed spectral relaxation is often lacking. The need for a complementary structural technique that is sensitive to changes in tertiary and quaternary structures is evident. From this point of view, $X$-ray scattering is a valuable tool. In X-ray scattering, the sample electron density $\rho(\mathbf{r})$ is related to the measured scattered intensity $I(\mathbf{q})$ via a Fourier transformation: $I(\mathbf{q})=|\mathbf{F T}[\rho(\mathbf{r})]|^{2}$, where $\mathbf{r}$ and $\mathbf{q}$ are the coordinates of real and reciprocal space, respectively. The magnitude of the scattering vector is $q=4 \pi / \lambda \sin (\theta / 2)$, where $\lambda$ is the $X$-ray wavelength, and $\theta$ is the scattering angle. This means that a characteristic length $D$ of the sample does not necessarily correspond to a single peak at $q=2 \pi / D$ unless long-range order is present $\uparrow$. Conversely, the signal at a single $q$-value does not correspond to a specific length scale. ${ }^{18} \mathrm{~A}$ clear advantage of X-ray scattering over spectroscopical techniques is the possibility of calculating the scattering pattern starting from the atomic coordinates of a given system. Several softwares that are able to calculate the scattering pattern of a protein in solution starting from crystallographic structures are available. ${ }^{19}$ Recently, we demonstrated that time-resolved wide-angle X-ray scattering (TRWAXS) can be used to accurately probe structural changes of proteins in solution with nanosecond time resolution. ${ }^{20}$ TR-WAXS combines the high time resolution important for biological samples with the high structural sensitivity of WAXS studies; it is therefore well suited for complementing information coming from time-resolved optical spectroscopy with more direct and global structural data. A first application of TR-WAXS to human $\mathrm{Hb}$ suggested that the timescale of the $\mathrm{R}-\mathrm{T}$ transition $\left(\tau_{\text {RT }}\right)$ is, in fact, $\sim 2 \mu$ s (i.e., about 1 order of magnitude faster than estimated with time-resolved optical spectroscopy). In view of the importance of this unexpected result, we have performed additional experiments to further investigate the $\mathrm{R}-\mathrm{T}$ transition of human $\mathrm{Hb}$ with TR-WAXS. In contrast to our first study, great care was taken to perform the experiments under conditions as close as possible to those used in most spectroscopic studies. In order to obtain a more accurate estimate of $\tau_{\mathrm{RT}}$, we probed the kinetics with an increased number of time points per decade. Moreover, we have examined the dependence of the observed kinetics on experimental parameters such as protein concentration, laser energy density, $\mathrm{pH}$, and buffer composition by performing TR-WAXS experiments under a variety of experimental conditions. The results of this investigation show clearly that the main quaternary rearrangement connected with the $\mathrm{R} \rightarrow \mathrm{T}$

$\uparrow$ For example, in the case of a multisubunit protein, even if the distance between subunits is equal to $D$, the resulting $I(\mathbf{q})$ would be spread over a large $q$-range roughly centered around $q=2 \pi / D$. 


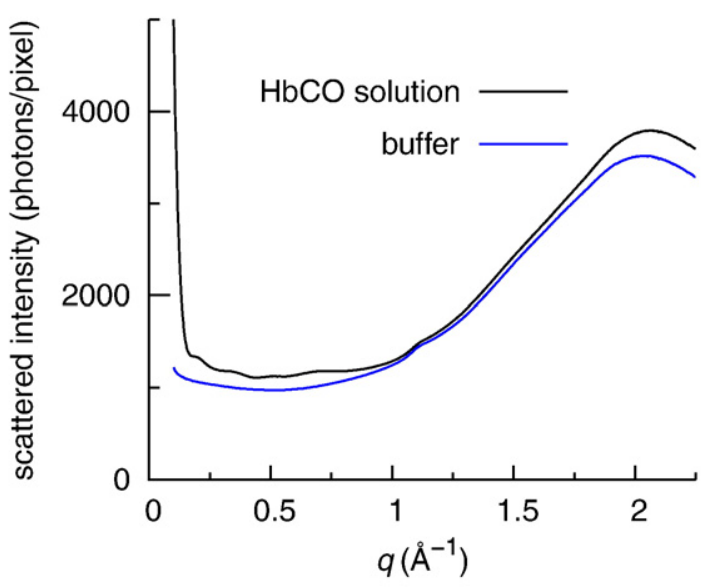

Fig. 1. Scattering pattern of a $0.5 \mathrm{mM} \mathrm{HbCO}$ solution contained in a quartz capillary having a diameter of $2 \mathrm{~mm}$, together with the scattering pattern of an identical quartz capillary filled with buffer.

transition occurs at physiological $\mathrm{pH}$ and room temperature at a timescale of about $2 \mu \mathrm{s}$.

\section{Results}

The basic idea of TR-WAXS is analogous to that of classical pump-probe spectroscopic techniques: a laser pulse is used to trigger a chemical reaction in the sample (ligand dissociation in the following), and a delayed X-ray pulse is used to probe induced structural changes. Scattered X-rays are collected by a two-dimensional detector and, typically, the magnitude of the scattering vector $\mathbf{q}$ ranges between $0.05 \AA^{-1}$ and $2.5 \AA^{-1}$.

Figure 1 shows the scattering pattern of a $0.5 \mathrm{mM}$ $\mathrm{HbCO}$ \% solution $(0.1 \mathrm{M}$ potassium phosphate buffer, $\mathrm{pH} 7$ ) contained in a 2-mm quartz capillary, together with the scattering pattern obtained from an analogous capillary filled with the same solvent. The protein contribution to the scattering pattern below $0.1 \AA^{-1}$ lacks information on structural details and is essentially related only to the protein average linear dimension $\S$. At higher $q$ values, a more structured pattern appears, mainly due to correlations among the protein structural motifs $\left(q<1.5 \AA^{-1}\right)$. The scattering of water dominates at higher $q$ values $\left(\sim 2 \AA^{-1}\right)$.

In TR-WAXS experiments, the scattering pattern measured before photoexcitation is always subtracted from the pattern measured at a given time delay after the laser pulse. In this way, the laserinduced signal is isolated from any non-laserdependent component of the signal. Figure 2 reports TR-WAXS difference patterns measured at several

$\$$ Here and in the following, protein concentration is expressed in millimolars of tetramers.

$\S$ It can be shown that for an "object" of typical dimension $D$, the scattering pattern for $q \leqslant 2 \pi / D$ can be approximated with a Gaussian function to a very high degree of fidelity, regardless of the details of the object. time delays after photolysis of a $0.5 \mathrm{mM} \mathrm{HbCO}$ solution at $20^{\circ} \mathrm{C}$ containing $0.1 \mathrm{M}$ phosphate buffer at $\mathrm{pH}$ 7. In the $q$-region where the protein signal dominates $\left(q<1.5 \AA^{-1}\right)$, the time evolution of these difference patterns indicates that the population of $\mathrm{Hb}$ molecules probed by the X-rays changes as a function of time. Liquid water molecules have an average distance of about $3 \AA$, giving rise to the peak at $2 \AA^{-1}$ in the scattering pattern (Fig. 1). Small changes in temperature (due to the laser energy absorbed by the protein and released to the solvent) slightly modify water distance distributions that in turn modify scattering around the water peak; this is the origin of the difference signal (heating signal) that contributes mainly to the $1.5-2.5 \AA^{-1} q$-region, as we have previously shown. ${ }^{20}$

TR-WAXS patterns have been collected at 37 different time delays between $147 \mathrm{~ns}$ and $300 \mathrm{~ms}$. The difference pattern measured at 147 ns after photolysis arises from the differences, mainly at the tertiary structural level, between the $\mathrm{Hb}$ transient structure $\left(\mathrm{Hb}^{*}\right)$ and the $\mathrm{HbCO}$ equilibrium structure. In view of the early appearance of such signal, we can exclude that it is due to a quaternary transition.

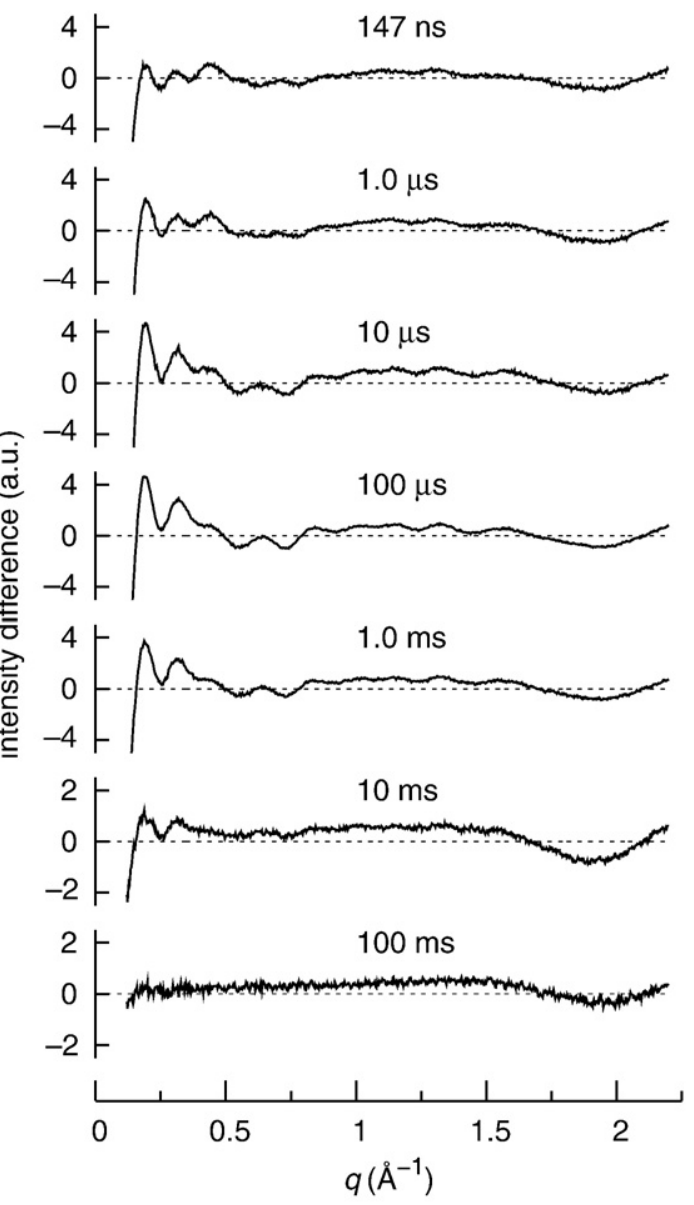

Fig. 2. TR-WAXS difference patterns (photolyzedunphotolyzed) measured after the photolysis of a $0.5 \mathrm{mM} \mathrm{HbCO}$ solution [0.1 M potassium phosphate buffer ( $\mathrm{pH} \mathrm{7)}$ and $8 \mathrm{mM}$ sodium dithionite] at $20{ }^{\circ} \mathrm{C}$. Data at selected time delays between $147 \mathrm{~ns}$ and $100 \mathrm{~ms}$ are shown. 


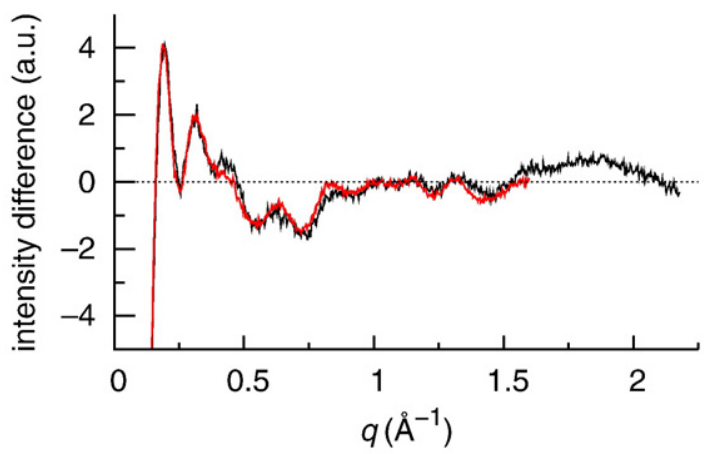

Fig. 3. Comparison of the TR-WAXS signal measured at $10 \mu \mathrm{s}$ from photolysis (black curve) with the difference between deoxyHb static pattern and $\mathrm{HbCO}$ static pattern. The contribution of the heating signal has been removed from the TR-WAXS pattern. The static patterns and the heating removal procedure are taken from Cammarata et al. ${ }^{20}$

Moreover, as we have previously shown, ${ }^{20}$ the main features of this signal can be modeled as the rotation of the E and F helices induced by heme doming, as suggested by Guallar et al. ${ }^{21}$ Further experimental evidence on the assignment of the 147-ns signal to tertiary relaxation is reported in Supporting Information. Between $1 \mu \mathrm{s}$ and $10 \mu \mathrm{s}$, the TR-WAXS signal changes in shape and grows in intensity. We have demonstrated that $\mathrm{Hb}$ molecules undergo the $\mathrm{R}-\mathrm{T}$ transition (tertiary and quaternary structural changes) in this timescale by comparing the $100-\mu \mathrm{s}$ TR-WAXS pattern with the difference between the deoxygenated $\mathrm{Hb}($ deoxyHb) static pattern and the $\mathrm{HbCO}$ static pattern. ${ }^{20}$ This conclusion was further confirmed by comparing the data with the difference signal calculated from crystallographic structures of $\mathrm{HbCO}$ and deoxyHb. ${ }^{20}$ For the sake of clarity, we report in Fig. 3 a comparison of the new
10- $\mu$ s pattern with the deoxy $\mathrm{Hb}-\mathrm{HbCO}$ static difference. Between $100 \mu \mathrm{s}$ and $10 \mathrm{~ms}$, the signal clearly loses intensity (for $q<1.0 \AA^{-1}$ ) without a significant change in shape as a result of $\mathrm{CO}$ rebinding to $\mathrm{Hb}$. Finally, at $100 \mathrm{~ms}$, an almost featureless signal is observed entirely due to solvent heating. ${ }^{22}$ Indeed, it is well known from time-resolved optical spectroscopy that $\mathrm{CO}$ rebinding to $\mathrm{Hb}$ is largely completed within tens of milliseconds.

The new data are in good agreement with our previous data in spite of the differences in sample preparation $(\mathrm{Hb}$ was not lyophilized in the present study), buffer composition, and $\mathrm{pH}$ (0.1 M potassium phosphate at $\mathrm{pH} 7$ versus $0.05 \mathrm{M}$ sodium phosphate at $\mathrm{pH}$ 7.4). The amplitude of the solvent heating signal is smaller in this study: this is mainly due to the smaller $\mathrm{Hb}$ concentration $(0.5 \mathrm{mM}$ versus $1.2 \mathrm{mM})$. Indeed, if the laser energy density is kept constant, the temperature increase in the solvent is, to a first approximation, proportional to the protein concentration. We stress that the experimental conditions chosen for the present study are as close as possible to those used in most spectroscopic studies (see, e.g., Jones et al. ${ }^{23}$ ).

While Fig. 2 satisfactorily depicts the most significant changes in the shape of TR-WAXS patterns, it does not explain the underlying structural changes. To better evaluate the latter throughout the 37 time delays investigated, we show the intensity difference in Fig. 4 for the $q$-value where the $\mathrm{Hb}$ contribution is biggest $\left(q \simeq 0.2 \AA^{-1}\right)$. Figure $4 \mathrm{~b}$ clearly shows that the " $\mathrm{Hb}^{\prime}$ " signal undergoes a rapid increase between $100 \mathrm{~ns}$ and $10 \mu \mathrm{s}$, stays almost constant between approximately $10 \mu \mathrm{s}$ and $100 \mu \mathrm{s}$, and later starts to decrease. We would like to point out that the amplitude of the TR-WAXS signal below $1.0 \AA^{-1}$ has reached its maximum value by $10 \mu \mathrm{s}$, indicating that the largest structural rearrangement (i.e., the $\alpha \beta$ dimers relative rotation) has already occurred. A rough estimation of the
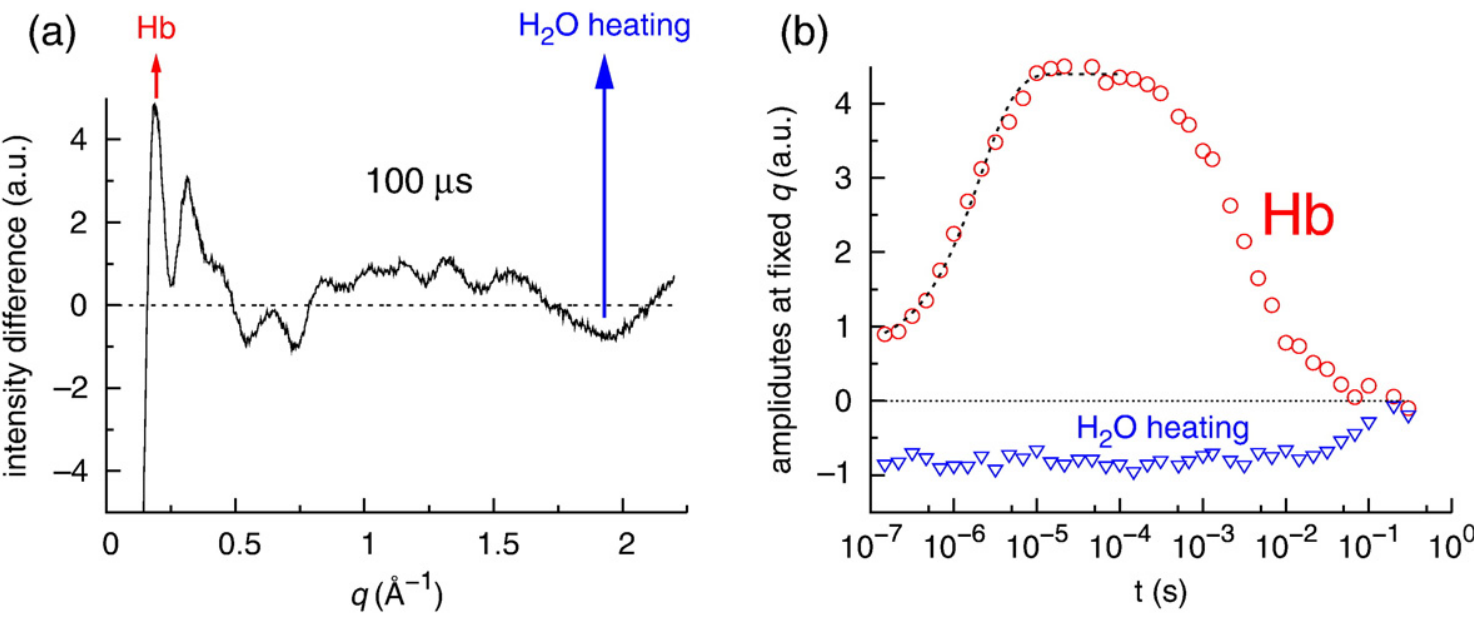

Fig. 4. Time dependence of the amplitude of the TR-WAXS patterns. (a) Data at $100 \mu$ s showing where the "Hb" and " $\mathrm{H}_{2} \mathrm{O}$ heating" signals have been selected for the plot in (b). (b) Intensity difference as a function of time delay obtained as the average of five $q$ points around the " $\mathrm{Hb}$ " and " $\mathrm{H}_{2} \mathrm{O}$ heating" positions indicated in (a). The black broken line is an exponential fit of the " $\mathrm{Hb}^{\prime}$ " signal for time delays shorter than $100 \mu$ s (see the text). 

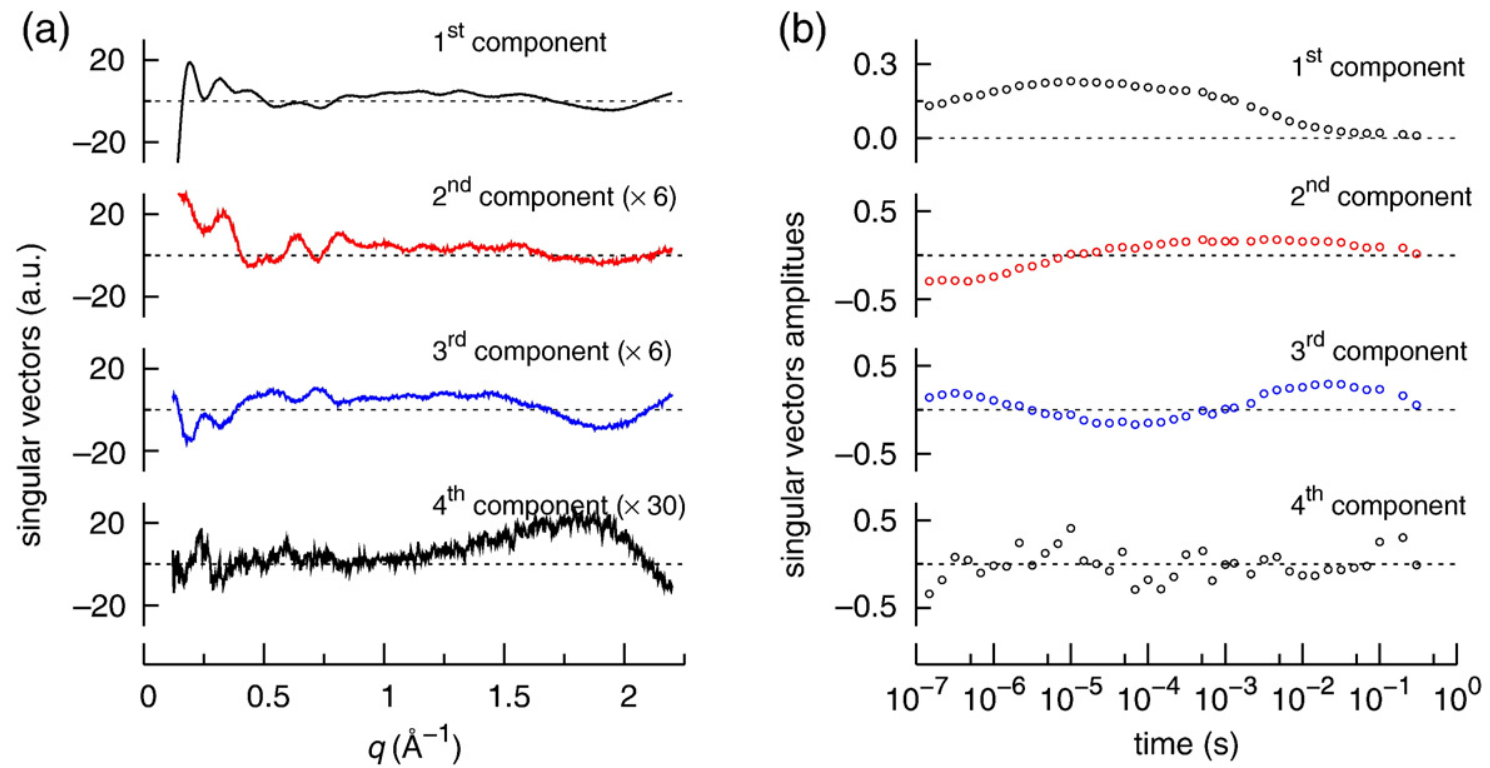

Fig. 5. (a) The four most significant SVD vectors of the collected TR-WAXS data [0.5 mM Hb and $0.1 \mathrm{M}$ phosphate buffer (pH 7)]. (b) Associated SVD amplitudes.

timescale characterizing this transition can be obtained by fitting the " $\mathrm{Hb}^{\prime}$ " signal below $100 \mu \mathrm{s}$ with an exponential rise (see broken curve in Fig. $4 \mathrm{~b})$, yielding a time constant of $2.1 \mu \mathrm{s}$. Note that such estimation does not take into account the complexity of the ligand rebinding process, and a full kinetic model is needed to extract the timescale of the R-T transition in a reliable way.

Raw data can also be used to estimate the time dependence of the transient temperature increase. In fact, for small values $\left(10^{\circ} \mathrm{C}\right)$, the difference signal due to solvent rearrangement is proportional (in amplitude) to the temperature difference. ${ }^{22}$ For $q \simeq$ $2 \AA^{-1}$, the total signal is dominated by the solvent (water) response, and the intensity difference is expected to be proportional to the temperature difference $\Delta T=T(t)-T(\infty)$. Figure $4 \mathrm{~b}$ shows that, as expected under our experimental conditions, ${ }^{24} \Delta T$ is constant up to time delays of $\sim 10 \mathrm{~ms}$ when heat diffusion starts to cool down the excited region.

In order to obtain a more in-depth picture of the phenomena occurring after photolysis, we have applied singular value decomposition (SVD) analysis ${ }^{25}$ in the $q$-range $0.13-2.2 \AA^{-1} \|$. SVD gives a series of $q$-patterns (singular vectors) and timedependent amplitudes (singular vector amplitudes) that are able to reproduce the data with increasingly high fidelity as the number of singular vectors retained is increased. The advantage of the SVD analysis is that it provides a model-independent estimation of the number of structurally distinguishable species. By evaluating the magnitude of the

II Although we have collected data in the range 0.05 $2.5 \AA^{-1}$, data between $2.2 \AA^{-1}$ and $2.5 \AA^{-1}$ are very noisy since they were measured using only the corners of a square CCD camera, while data between $0.05 \AA^{-1}$ and $0.13 \AA^{-1}$ are affected by residual slit scattering. singular vectors, their $q$-dependence, and the time dependence of the scattering vector amplitudes, we concluded that our data are well reproduced, within the signal-to-noise ratio, by the first three singular components (Fig. 5a and b). Indeed, the fourth component, apart from being significantly smaller than the first three components, is characterized by an amplitude varying randomly with time. We have verified in separate experiments that this component is mainly due to the random vibrations of the capillary containing the sample. The relatively small number of independent components is surprising. With $\mathrm{Hb}$ being a tetramer, one would expect different ligation states in the $\mathrm{R}$ and $\mathrm{T}$ quaternary conformations to be present in solution, thus bringing about a high level of structural heterogeneity. This expectation is not supported by the data within the present signal-to-noise ratio.

SVD analysis is very useful in providing a minimal description of the experimental data without any loss of information content. ${ }^{26,27}$ However, the SVD components do not have, in the most general case, a direct physical meaning. For elucidation of the reaction pathway that leads to the quaternary $\mathrm{R}-\mathrm{T}$ transition, it is thus necessary to make a kinetic model of the reaction pathway in terms of interconversion between different molecular species. Furthermore, a mathematical relation between the molecular species actually existing in solution and the observed TR-WAXS patterns has to be established. To this purpose, we have followed an approach used for analyzing time-resolved spectroscopic data. ${ }^{28,29}$ The basic idea is to add a priori information on kinetics to obtain a minimal set of $q$-patterns (basis patterns) that are able to reproduce the data and, at the same time, have a clear physical meaning. The relevant physical states in this model are the ligation states of the two $\mathrm{Hb}$ quaternary 
structures: $T_{i}$ and $R_{i}$ (where $i=0-4$ indicates the number of bound ligands). The kinetic model, schematically represented in Fig. 6, corresponds to a set of differential equations: one for each of these states, plus one describing the evolution of the solution temperature. In view of the SVD result, we have tried to decompose our TR-WAXS patterns in terms of three different basis patterns:

$$
\begin{aligned}
\Delta S(q, t)= & R_{\text {like }}(t) \Delta S_{R_{\text {like }}}(q)+T_{\text {like }}(t) \Delta S_{T_{\text {like }}}(q) \\
& +\Delta T(t) \Delta S_{\mathrm{H}}(q)
\end{aligned}
$$

where $\Delta S_{R_{\text {like }}}(q)$ is the basis pattern corresponding to the tertiary relaxations occurring within the $\mathrm{R}$ states, $\Delta S_{T_{\text {like }}}(q)$ is the basis pattern corresponding to the R$\mathrm{T}$ transition, and $\Delta S_{\mathrm{H}}(q)$ is the basis pattern corresponding to solvent heating. Accordingly, $R_{\text {like }}(t)$ is a weighted sum of the $R_{i}(t)$ populations, and $T_{\text {like }}(t)$ is a weighted sum of the $T_{i}(t)$ populations, while $\Delta T(t)$ is equal to the temperature jump at time $t$ from photolysis. The kinetic parameters featured in the model (see Materials and Methods) and the basis spectra have been fitted against the experimental TR-WAXS patterns. ${ }^{29}$ Note that this approach assumes that the high structural heterogeneity expected does not affect the data, as shown by the SVD analysis.

The kinetic model used to fit the evolution of populations is similar to the one proposed by Sawicki and Gibson to analyze flash photolysis data at comparable time delays. ${ }^{6}$ Basically, it takes into account the bimolecular recombination of the $\mathrm{R}$ and $\mathrm{T}$ conformations and transitions between the two quaternary structures with the same ligation state. It also takes into account the time evolution of the solvent temperature due to heat diffusion. The explicit differential equations can be found in Supporting Information. The parameter $L$ regulates the equilibrium between the two quaternary structures in the absence of ligands (so-called allosteric constant), the parameter $c$ controls ligand affinity, and $s$ is introduced to take into account the proposed slowing of the quaternary transition rate with increasing number of bound ligands. ${ }^{6,29} D_{\mathrm{R}}$ and $D_{\mathrm{T}}$ are the microscopic bimolecular rebinding rates to the $\mathrm{R}$ and $\mathrm{T}$ states, respectively. To solve the set of differential equations corresponding to the model of Fig. 6, we used the following initial conditions: $T_{i}=0$ and $R_{i}=\left(\begin{array}{c}i \\ 4\end{array}\right) N_{0}^{i}\left(1-N_{0}\right)^{4-i}$, where $N_{0}$ is the fraction of deoxyhemes after the pump pulse. The choice of the $R_{i}$ initial populations is justified by the assumption that each photolysis event is uncorrelated with the previous photolysis of a heme-CO complex of the same $\mathrm{Hb}$ tetramer. In this hypothesis, the distribution of ligated species right after photolysis is given by the above binomial distribution of $R_{i}$. With the above assumptions, the resulting kinetic model has seven independent parameters $\left(K, L, c, S, D_{R}\right.$, $D_{\mathrm{T}}$, and $N_{0}$ ). In order to reduce the number of
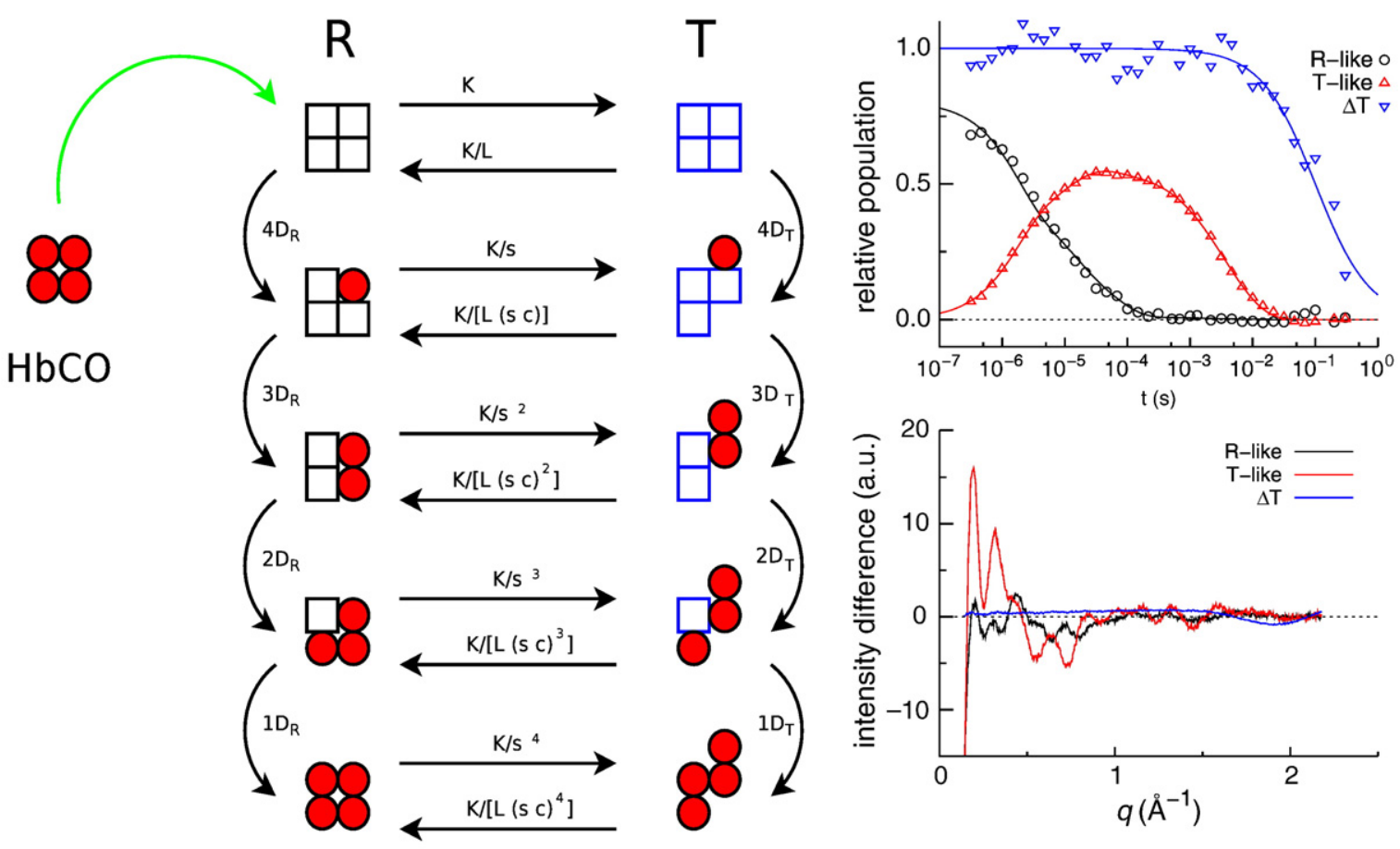

Fig. 6. Left: Scheme of the two-state kinetic model used for data analysis. Right, top: Time dependence of $R_{\text {like, }} T_{\text {like, }}$ and $\Delta T$ as calculated from the best-fit parameters [the sample is composed of $0.5 \mathrm{mM} \mathrm{Hb}$ and $0.1 \mathrm{M}$ phosphate buffer ( $\mathrm{pH}$ 7)]. Right, bottom: Corresponding basis spectra. $D_{\mathrm{R}}$ and $D_{\mathrm{T}}$ are the bimolecular rebinding rates for the $\mathrm{R}$ and $\mathrm{T}$ species, respectively; $K$ is the rate of the $R_{0}-T_{0}$ transition; $L$ is the $\left[T_{0}\right] /\left[R_{0}\right]$ ratio; $c$ is the $K_{\mathrm{T}} / K_{\mathrm{R}}$ ratio, where $K_{\mathrm{R}}$ and $K_{\mathrm{T}}$ are the equilibrium association constants to $\mathrm{R}$ and $\mathrm{T}$ species, respectively; and $s$ is a factor that takes into account the slowing of the R-T transition with increasing number of bound ligands. 


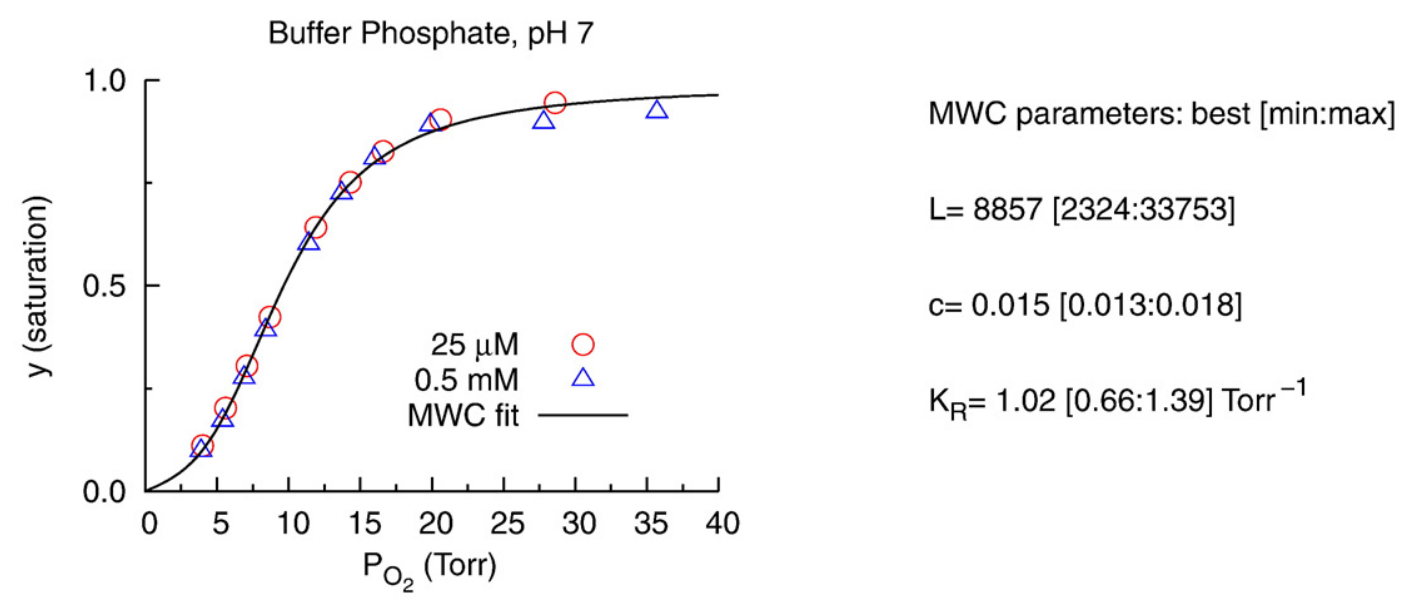

Fig. 7. Oxygen saturation curve of two samples analogous to that used for TR-WAXS experiments ( $0.1 \mathrm{M}$ phosphate buffer, $\mathrm{pH} 7$ ) having $\mathrm{Hb}$ concentrations of $0.025 \mathrm{mM}$ and $0.5 \mathrm{mM}$, respectively. The black line is a fit of the data in terms of the Monod-Wyman-Changeux model. ${ }^{4}$ The parameter $L$ has already been defined in the main text, and $c$ is the $K_{\mathrm{T}} / K_{\mathrm{R}}$ ratio, where $K_{\mathrm{R}}$ and $K_{\mathrm{T}}$ are the equilibrium association constants of oxygen to R-state and $\mathrm{T}$-state $\mathrm{Hb}$, respectively.

fitting parameters, we have experimentally fixed the $L$ value by measuring the oxygen equilibrium curve of an $\mathrm{Hb}$ sample almost identical with that used for TR-WAXS experiments (Fig. 7). The quality of the fit of the TR-WAXS data can be estimated by a reduced global $\chi^{2}$, as discussed in Materials and Methods, but also by deconvolving the experimental signal measured at each time delay as a linear combination of the basis spectra found during the fit procedure. Excellent agreement is obtained, as shown in Fig. 8 for selected time delays, together with the residuals shown in a 10-fold expanded scale. The time dependence of the three components $R_{\text {like, }} T_{\text {like, }}$ and $\Delta T$ is shown in Fig. 6 . The time dependence of $T_{\text {like }}$ and $\Delta T$ is close to that estimated from the raw data as reported in Fig. 4 . The points nicely follow the predicted behavior, thus suggesting that the kinetic model is able to reproduce the data with high fidelity.

Note that in the two-state kinetic model used to fit our TR-WAXS data, it is assumed that the transition from the fast bimolecular $\mathrm{CO}$ rebinding rate to the slow one occurs simultaneously with the main quaternary structure change. This last assumption is by no means proven by our data. Indeed, it is possible to obtain good fits even if the switch between the fast $\mathrm{CO}$-recombining species and the slow CO-recombining species occurs in a later step with respect to the $\mathrm{Hb}$ dimers rotation (see Supporting Information). Thus, a small structural change, likely localized around the heme and barely detectable by TR-WAXS, could induce the switch from $D_{\mathrm{R}}$ to $D_{\mathrm{T}}$, in accordance with the early observation by Sawicki and Gibson. ${ }^{6}$ We have refrained from reporting a kinetic model implementing such possibility, since this involves a larger number of accessible states and fitting parameters. The simplicity of the reported model is able to accurately reproduce the kinetic processes observed with TRWAXS in terms of physical parameters easily comparable with those usually reported in the literature. We stress that even if our TR-WAXS data are not directly sensitive to the change in the
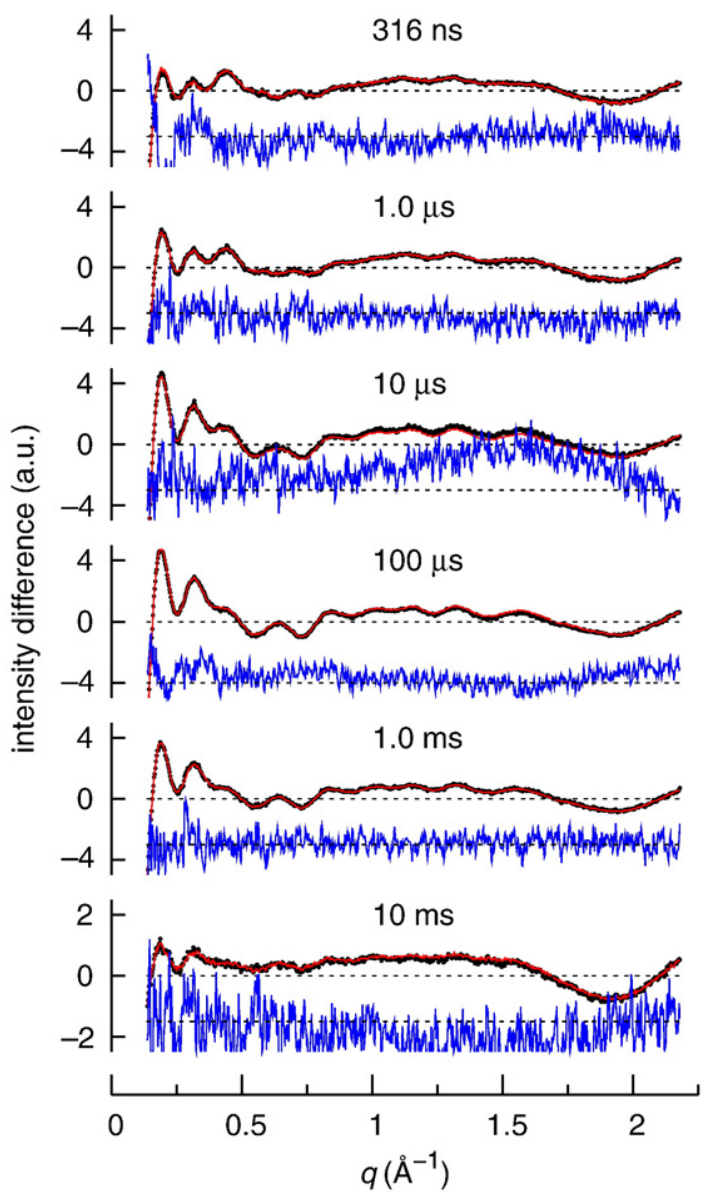

Fig. 8. Data at selected time delays from photolysis [0.5 $\mathrm{mM} \mathrm{Hb}$ and $0.1 \mathrm{M}$ phosphate buffer ( $\mathrm{pH} 7$ )], together with the corresponding fits obtained with the kinetic model presented in Fig. 6. Residuals are reported in an expanded scale. 
Table 1. Parameters obtained by the fitting procedure described in the text, in parentheses (minimum:maximum)

\begin{tabular}{|c|c|c|c|c|c|}
\hline & $\begin{array}{c}\text { Hepes (pH 7) } \\
\text { and } 0.57 \mathrm{mM} \mathrm{Hb}\end{array}$ & $\begin{array}{l}\text { Hepes (pH 8.5) } \\
\text { and } 0.70 \mathrm{mM} \mathrm{Hb}\end{array}$ & $\begin{array}{c}\text { K-pho (pH 7) } \\
\text { and } 0.25 \mathrm{mM} \mathrm{Hb}\end{array}$ & $\begin{array}{c}\text { K-pho (pH 7) } \\
\text { and } 0.50 \mathrm{mM} \mathrm{Hb}\end{array}$ & $\begin{array}{c}\text { K-pho (pH 7) } \\
\text { and } 1.00 \mathrm{mM} \mathrm{Hb}\end{array}$ \\
\hline$N_{0}$ & $0.86(0.85: 0.86)$ & 0.85 & $0.76(0.75: 0.77)$ & $0.80(0.80: 0.81)$ & $0.72(0.70: 0.73)$ \\
\hline$\tau_{\mathrm{RT}}(\mu \mathrm{s})$ & $1.8(1.7: 1.8)$ & 70.7 (64.6:77.8) & $1.9(1.8: 2.1)$ & $1.9(1.8: 2.0)$ & $1.2(1.1: 1.3)$ \\
\hline$L\left(\times 10^{-3}\right)$ & 1.38 & 1.48 & 8.85 & 8.85 & 8.85 \\
\hline$c\left(\times 10^{3}\right)$ & $7.57(6.76: 8.47)$ & $0.08(0.07: 0.09)$ & $0.81(0.63: 1.03)$ & $2.92(2.63: 3.23)$ & $6.14(5.15: 7.13)$ \\
\hline$s$ & $31(28: 33)$ & 20 & $26(24: 30)$ & $14(13: 14)$ & $15(14: 16)$ \\
\hline$D_{\mathrm{R}}\left(\mu \mathrm{M}^{-1} \mathrm{~s}^{-1}\right)$ & $6.7(6.4: 7.0)$ & 7.0 & $8.7(8.0: 9.4)$ & $7.9(7.6: 8.2)$ & 14.3 (13.0:15.6) \\
\hline$D_{\mathrm{T}}\left(\mu \mathrm{M}^{-1} \mathrm{~s}^{-1}\right)$ & 0.039 (0.038:0.040) & 0.045 & $0.037(0.036: 0.039)$ & $0.031(0.030: 0.032)$ & $0.059(0.057: 0.062)$ \\
\hline$\Delta T(\mathrm{~K})$ & 0.7 & 0.7 & 0.4 & 1.0 & 2.0 \\
\hline
\end{tabular}

microscopic CO rebinding rate from "fast" to "slow," they unambiguously set the timescale of the main structural transition to $2 \mu \mathrm{s}$ (see Supporting Information).

Recently, Makowski et al. have observed that the structure of $\mathrm{Hb}$ is a function of concentration below $\simeq 1 \mathrm{mM}^{30}$ To verify any dependence of the $\mathrm{R} \rightarrow \mathrm{T}$ transition timescale on protein concentration, we have performed experiments at three different $\mathrm{Hb}$ concentrations: $0.25 \mathrm{mM}, 0.5 \mathrm{mM}$, and $1.0 \mathrm{mM}$. Fits of similar quality have been obtained at the different $\mathrm{Hb}$ concentrations; the best-fit parameters are reported in Table 1. As further control and in order to examine the dependence of the observed kinetics on buffer composition and $\mathrm{pH}$, we have also performed experiments in Hepes buffer at $\mathrm{pH} 7.4$ and $\mathrm{pH} 8.5$, at the constant protein concentration of $\sim 0.6 \mathrm{mM}$. Typical examples of TR-WAXS patterns measured for these samples are reported in Supporting Information, together with details on data analysis.

\section{Discussion}

The main result of this work is that for all samples at $\mathrm{pH} 7$, the value of the parameter $\tau_{\mathrm{RT}}$ (i.e., the time constant of the $R_{0} \rightarrow T_{0}$ quaternary transition) is about $2 \mu \mathrm{s}$, almost independent of buffer composition and protein concentration (and of the temperature jump that in the present experiments is on the order of only $1-2{ }^{\circ} \mathrm{C}$; see Table 1 ). The fact that the experiment with $1 \mathrm{mM} \mathrm{Hb}$ gives a slightly smaller $\tau_{\mathrm{RT}}$ value (1.2 $\mu \mathrm{s}$ compared to $\left.1.9 \mu \mathrm{s}\right)$ might be indicative of a small temperature effect (corresponding to a $\mathrm{R} \rightarrow \mathrm{T}$ activation enthalpy barrier on the order of $40 \mathrm{kcal} \mathrm{mol}^{-1}$ ). On the other hand, the fact that the $\tau_{\text {RT }}$ value measured in Hepes buffer at $\mathrm{pH} 7$ is close to that measured in phosphate buffer is in line with the action of phosphate buffer as a weak allosteric effector that binds to and stabilizes T-state $\mathrm{Hb}$ (as evidenced by the higher $L$ value; see Table 1) but barely binds to $\mathrm{Hb}$ in $\mathrm{R}$ conformation. Corroborating evidence comes from the experiment at $\mathrm{pH} 8.5$, where the substantially larger $\tau_{\mathrm{RT}}$ value of $70 \mu$ s is measured, in agreement with expectations. ${ }^{6}$

The data reported in Table 1 deserve further comments. First of all, we note the overall good agreement of our parameter values with similar data in the literature. In particular, the CO bimolecular rebinding constants to $\mathrm{R}$-state and $\mathrm{T}$-state $\mathrm{Hb}$ are in good agreement with those obtained with timeresolved spectroscopy. ${ }^{17,23}$ Parameter $N_{0}$, which represents the fraction of deoxyhemes right after the photolysis pulse, ranges from 0.72 to 0.86 (i.e., it is substantially higher than that observed by Jones et al. at similar time delays using 10-ns laser pulses ${ }^{23}$ ). This is not surprising since the "long" (230 ns) laser pulse employed in this study ensures multiple photolysis of the sample. As already noted, the presence of a slow geminate phase at $\sim 100 \mathrm{~ns}^{31}$ limits the efficiency of multiple photolysis, so that $N_{0}$ values of 1 are not obtained in our experiments. Concerning the $s$ parameter (i.e., the slowing of the quaternary transition rate with ligation number), we have obtained values of $\sim 15$ (i.e., larger than what has been obtained with time-resolved spectroscopy; $s \sim 5) .{ }^{6,28}$ To further investigate this point, we have performed a constrained fitting of the $0.5 \mathrm{mM} \mathrm{Hb}$ data in which $s$ was fixed to 5 : this yielded a $\tau_{\mathrm{RT}}$ value of $3 \mu \mathrm{s}$ (compared to $\tau_{\mathrm{RT}}=1.9 \mu \mathrm{s}$ obtained in the unprejudiced fittings) with a $2 \%$ increase in $x^{2}$. The obtained $s$ value corresponds to a time constant for the $R_{1} \rightarrow T_{1}$ transition of about 20-30 $\mu$ s; however, we note that using a three-state kinetic model to fit our data (see Supporting Information), we can obtain smaller $s$ values consistent with those deduced from time-resolved spectroscopical evidence.

As mentioned in the Introduction, the "classical" spectroscopic picture of the structural events occurring in $\mathrm{Hb}$ after flash photolysis of the bound $\mathrm{CO}$ involves (besides geminate rebinding) a series of submicrosecond events followed by a $\sim 20-\mu \mathrm{s}$ relaxation, usually attributed to the $R_{0} \rightarrow T_{0}$ quaternary transition, and by bimolecular rebinding on the milliseconds timescale. In our TR-WAXS experiment, we see an "early transition signal" fully developed already at $300 \mathrm{~ns}$, followed in the time range $300 \mathrm{~ns}-100 \mu \mathrm{s}$ by only one relaxation occurring at about $2 \mu \mathrm{s}$. In the picture emerging from the TR-WAXS studies, a tertiary relaxation-consistent with rotation and translation of the E and F helices' clamshell $^{20,21,32}$ and occurring earlier than $200 \mathrm{~ns}-$ is followed by a main quaternary relaxation likely involving the $\alpha \beta$ dimers relative rotation and translation and occurring in a concerted way at about $2 \mu \mathrm{s}$. With the present TR-WAXS accuracy, nothing is seen at about $20 \mu \mathrm{s}$, where the spectroscopic studies identify a relaxation usually attributed to the $R_{0} \rightarrow T_{0}$ quaternary transition. There is not necessarily a contradiction between TR-WAXS and optical spectroscopy data: the $20-\mu$ s relaxation likely 
corresponds to small and localized structural changes not detectable with the present TR-WAXS signal-to-noise ratio and occurring later than the main (quaternary) structural rearrangement. This 20- s WAXS-silent relaxation could be responsible for the transition from "fast-rebinding" $\mathrm{Hb}$ to "slowrebinding" $\mathrm{Hb}$ (see Supporting Information).

We would like to stress the agreement of our picture with that emerging from the TR-UVRR studies by Jayaraman et al. ${ }^{13}$ and Balakrishnan et $a .^{33}$ whereby fast submicrosecond relaxations (attributed to the rotation/translation of the E and F helices' clamshell and to the breaking/reformation of interhelical hydrogen bonds) undetected in our TR-WAXS experiments are followed by a $\sim 3-\mu \mathrm{s}$ relaxation that the authors attribute to T-state contact formation in the hinge region and dimer rotation.

In our opinion, one point remains surprising: we are able to account for the entire time course of our difference signals using only two basis patterns (see Fig. 5) in spite of the fact that partially ligated states $\left(R_{i}\right.$ and $T_{i}$, where $\left.i=0-4\right)$ are expected to be significantly populated under our experimental conditions. This means that within the signal-tonoise ratio of our data, $\mathrm{Hb}$ molecules that differ only in the number of bound ligands are almost indistinguishable. In fact, TR-WAXS enabled us to detect only two $\mathrm{Hb}$ conformations that are structurally different from the $\mathrm{HbCO}$ conformation and have been interpreted as the $\mathrm{T}$ state and a tertiary relaxed form of the $\mathrm{R}$ state. This point calls for more accurate investigations combining structural and functional experimental techniques.

\section{Materials and Methods}

\section{$\mathrm{Hb}$ preparation}

Erythrocytes from freshly drawn heparinized human blood were washed four or five times with $0.9 \% \mathrm{NaCl}$ and then hemolyzed with pure peroxide-free ethyl ether. The stroma was precipitated with $\mathrm{NaCl}(10 \%$ by weight) and removed by centrifugation. The hemolysate was dialyzed overnight against $0.1 \mathrm{M}$ potassium phosphate buffer ( $\mathrm{pH} 7$ ) and then stripped of organic phosphates by passage through a Sephadex G-25 (fine) column equilibrated with $0.1 \mathrm{M}$ potassium phosphate. All steps were carried out at $4{ }^{\circ} \mathrm{C}$. The resulting $\mathrm{Hb}$ solution had a typical concentration of $\sim 12 \% \mathrm{wt} / \mathrm{vol}$. Stock solutions were stored as beads in liquid nitrogen with the protein in oxygenated form.

\section{Phosphate buffer samples}

The $\mathrm{Hb}$ stock solution was diluted with $0.1 \mathrm{M}$ potassium phosphate buffer ( $\mathrm{pH} 7$ ) to obtain a $1 \mathrm{mM} \mathrm{Hb}$ solution. The resulting solution was equilibrated for $\sim 20$ min with humidified CO. A small quantity of a $\mathrm{CO}$-saturated $\mathrm{Na}$-dithionite concentrated solution was added anaerobically to ensure full reduction of the iron. Samples at lower concentrations were prepared by further dilutions with CO-saturated buffer. The final Na-dithionite concentration was $8 \mathrm{mM}$ in all samples.
Approximately $100 \mu \mathrm{l}$ of a freshly prepared $\mathrm{HbCO}$ solution was loaded into X-ray quartz capillaries (Hilgenberg $\mathrm{GmbH}$ ) with a diameter of $2 \mathrm{~mm}$. Two CO bubbles were trapped on both sides of the protein solution, with a $\mathrm{CO}$-saturated glycerol drop preventing gas leakage at the open side of the capillary (see Supporting Information). The capillaries were further sealed with capillary wax (Hampton Research) and Epoxy glue.

\section{Hepes buffer samples}

Hepes ( $p H$ 7.4). Samples in Hepes buffer were prepared starting from the stock solution mentioned above. After proper dilution, the solution was dialyzed overnight against $0.1 \mathrm{M}(\mathrm{pH}$ 7.4) Hepes buffer. About $1 \mathrm{ml}$ of such solution was left for more than $30 \mathrm{~min}$ under $\mathrm{CO}$ flux while the solution was gently stirred. After addition of $1.4 \mathrm{mg}$ of $\mathrm{Na}$-dithionite and $30 \mathrm{~min}$ more of $\mathrm{CO}$ fluxing, a spectrum of a properly diluted sample confirmed sample quality, no detectable presence of met, and full CO saturation. $\mathrm{An} \mathrm{Hb}$ concentration of $0.57 \mathrm{mM}$ was calculated from the optical spectrum. Hepes ( $p H$ 8.5). The same procedure described above was performed using $0.1 \mathrm{M}$ Hepes buffer ( $\mathrm{pH} 9$ ). The final sample concentration and $\mathrm{pH}$ value were measured to be $0.7 \mathrm{mM}$ and 8.5 , respectively.

\section{Sample verification}

Prior to each experiment, a few microliters of $\mathrm{Hb}$ solution were diluted in CO-saturated buffer and placed onto a 1-mm quartz cuvette to ensure that no significant fraction of methemoglobin $(\operatorname{metHb})$ was present. At the end of a long data collection, the capillaries were broken, and the solution was diluted in CO-saturated buffer to verify the status of the sample. A small fraction (always less than $5 \%$ ) of metHb was observed.

\section{TR-WAXS data collection}

TR-WAXS patterns were acquired at the ID9B beamline of the European Synchrotron Radiation Facility (Grenoble, France) while the machine was running in four-bunch mode. HbCO samples were photolyzed with a circularly polarized 527-nm laser pulse at a time duration of $230 \mathrm{~ns}$ (full width at half maximum). Different energy densities were used as a function of the $\mathrm{Hb}$ concentration. In the case of the $0.5 \mathrm{mM} \mathrm{Hb}$ sample, an energy density of $2.5 \mathrm{~mJ}$ $\mathrm{mm}^{-2}$ (at the capillary surface) was used. For the $0.25 \mathrm{mM}$ and $1.0 \mathrm{mM} \mathrm{Hb}$ samples, proportionally lower and higher photolysis energy densities were used. Laser pulses incident on the top surface of the capillary were followed by delayed quasi-monochromatic X-ray pulses (100 ps, full width at half maximum) extracted from the synchrotron by means of a high-speed chopper and a millisecond shutter. ${ }^{34}$ X-ray pulses penetrated the capillary $0.2 \mathrm{~mm}$ below its top edge so that an orthogonal geometry between X-ray and photolysis pulses was realized. Scattering patterns at six time delays per decade between $147 \mathrm{~ns}$ and $300 \mathrm{~ms}$ were acquired. For time delays longer than $32 \mu \mathrm{s}$, seven X-rays pulses (i.e., 5- $\mu$ s-long pulse envelopes) were allowed to go through the chopper at each opening. No measurable difference at $32 \mu$ s could be noted between the data collected using the single pulse and the data collected using the seven pulses. For delays longer than $100 \mu \mathrm{s}, 15$ pulses $(\sim 10 \mu \mathrm{s})$ were used. The sample was maintained at $22{ }^{\circ} \mathrm{C}$ with a cold nitrogen 
stream (Oxford Cryostream). To dilute any X-ray radiation damage over a large sample volume, we translated the sample back and forth along its long axis over a 25-mm range. To ensure that successive pulses in the $2-\mathrm{Hz}$ to $10-\mathrm{Hz}$ pulse train excited adjacent but spatially separated sample volumes, we translated the capillary by $0.35 \mathrm{~mm}$ after each probe pulse. Scattered Xrays were recorded in the forward direction by a sensitive CCD camera (Mar133; Mar Research). Each image was azimuthally averaged and converted into a one-dimensional $q$-curve using $\lambda=0.6793 \AA$ corresponding to the peak of the X-ray spectrum. After normalization (in the $q$ region 2-2.2 $\AA^{-1}$ ), a reference scattering pattern ("laseroff " image), which probed the unexcited sample, was subtracted from the scattering pattern at a given time delay. Intensity differences calculated for the same time delay were averaged.

\section{SVD analysis}

SVD analysis starts by organizing a data set in an $n_{q} \times n_{t}$ matrix $M$ with $n_{q} q$-points and $n_{t}$ time delays. In our case, $n_{q}=857$ and $n_{t}=36 \uparrow$. The $n_{q} \times n_{t}$ matrix $M$ is expressed as $M=U \times S \times V^{\mathrm{T}}$, where $U$ is $n_{q} \times n_{t}$, with $U^{\mathrm{T}} \times U$ equaling the identity; $S$ is an $n_{t} \times n_{t}$ diagonal matrix; and $V$ is an $n_{t} \times n_{t}$ orthogonal matrix. Here superscript $\mathrm{T}$ indicates matrix transposition. $U$ contains $n_{t} q$ spectra that are orthonormal (i.e., $\sum_{k} U_{i, k} \cdot U_{k, j}=\delta_{i, j}$, where $\delta_{i, j}$ is the Kronecker delta). The diagonal matrix $S$ contains $n_{t}$ singular values that express the weight of each $q$ spectra found in $U$. The columns of the $U \times S$ matrix are named "singular vectors" in the main text and are ordered with decreasing norm. The matrix $V$ contains the amplitudes of the singular vectors for each time delay. The SVD analysis was performed using the library "newmat" (version 11) freely available on the Internet.

\section{Kinetic model for TR-WAXS data analysis}

The presence of a slower geminate phase $(\sim 100 \mathrm{~ns})^{31}$ limits the efficiency of multiple excitation when using a $\sim 230$-ns laser pulse. The temperature jump due to the laser excitation, for the timescales relevant to this study, can be assumed to vary according to the law $\Delta T(t)=\frac{\Delta T_{0}}{1+t / \tau_{\mathrm{T}}}$, where $\tau_{\mathrm{T}}$ is a parameter related to the thermal diffusion coefficient and laser beam size. ${ }^{24}$ The amplitude of the solvent response is assumed to be proportional to $\Delta T$. As discussed by Cammarata et al., the linear combinations used to represent the experimental signal are: $T_{\text {like }}(t)=$ $\sum_{i=0,4} T_{i}(t)$ and $R_{\text {like }}(t)=\sum_{i=0,4}(4-i) / 4 R_{i}(t)$ and $\Delta T^{20}$ It should be noted that while the $T_{i}$ values are simply summed (thus assuming that a molecule in $T_{0}$ would contribute to the scattering as any other molecule in the $\mathrm{T}$ quaternary state), the $R_{i}$ values have been rescaled according to the number of deoxyhemes. The reason for this choice is motivated by the fact that the structural origin of the differences is at the tertiary level; thus, they are expected to scale with the number of unbound subunits.

Although an SVD analysis can reveal the number of independent components necessary to describe the data (within the signal-to-noise ratio), the main limitation is

T To avoid any pump dynamics from affecting the data, we performed the fits without the 147-ns time delay data. that the singular vectors have no physical meaning. To overcome such limitation, several authors have used an SVD-like approach that returns meaningful $q$ spectra at the expense of defining a kinetic model for the population. A system of (coupled) differential equations is solved for the given initial conditions and a set of parameters that describe the kinetics; a linear combination of the solutions is taken to obtain $n_{p}$ functions that are the population of distinguishable species according to the experimental technique used (in our case proteins conformations with roughly the same structure). Calculating such functions for the time points experimentally measured allows us to organize the information in an $n_{t} \times n_{p}$ "population matrix" $P$. We define the $n_{b} \times n_{q}$ basis spectra matrix $B$ with the following system of linear equations:

$$
P \times B=M^{\mathrm{T}}
$$

The system described above is a collection of $n_{q} \times n_{t}$ equations with "only" $n_{q} \times n_{b}$ variables. When $n_{b}<n_{t}$, as is usually the case, the system is overdetermined, and the best-fit solution can be found by inverting Eq. (1):

$$
B=\left(P^{\mathrm{T}} \times P\right)^{-1} \times P^{\mathrm{T}} \times M^{\mathrm{T}}
$$

Once the $B$ matrix has been found using Eq. (2), a measure of "fit quality" can be obtained using the following $x^{2}$ :

$$
\chi^{2}=\sum_{i, j} \frac{(P \times B)_{i, j}-M_{i, j}}{\sigma_{i, j}}
$$

The fitting procedure can be integrated into a nonlinear minimization scheme by varying the parameters that control the system of differential equations for the populations of the different species. The full system of equation is reported in Supporting Information. The reduced $\chi^{2}$ is defined as $\chi_{0}^{2}=\chi^{2} /\left[n_{q}\left(n_{t}-n_{b}\right)-N_{\text {par }}\right]$, where $N_{\text {par }}$ is the number of free parameters that control the populations ( $N_{\text {par }}=7$ in our case).

\section{Oxygen binding curves}

Oxygen equilibrium curves of $\mathrm{Hb}$ in $0.1 \mathrm{M}$ potassium phosphate $(\mathrm{pH} 7)$ were determined with standard spectrophotometric/tonometric methods ${ }^{35}$ at two different $\mathrm{Hb}$ concentrations: $25 \mu \mathrm{M}$ and $0.5 \mathrm{mM}$ (in tetramers), respectively. These samples were identical with those used for TR-WAXS measurements, except that a small amount $(0.05 \%$ by weight) of Na-ethylenediaminetetraacetic acid was added to reduce the oxidation of the protein to the metHb form. After equilibration, the absorption spectrum of the sample was recorded in the desired wavelength range (470-670 $\mathrm{nm}$ or $600-830 \mathrm{~nm}$, depending on the $\mathrm{Hb}$ concentration used). Complete oxygenation of the sample was achieved by opening the tonometer and exposing the sample to air at atmospheric pressure. A more detailed description of the procedure and data analysis can be found in Supporting Information.

\section{Acknowledgements}

The authors would like to thank Philip Anfinrud, Friedrich Schotte (National Institutes of Health, USA), and Harry Ihee (Korea Advanced 
Institute of Science and Technology, South Korea) for useful discussions; and Giorgio Schirò, Alessandro Spilotros (University of Palermo, Italy), and Beatrice Vallone (University of Rome "La Sapienza", Italy) for help during the experiments. M.C. also acknowledges Martin Nielsen and the Center for Molecular Movie (University of Copenhagen) for support and funding.

\section{Supplementary Data}

Supplementary data associated with this article can be found, in the online version, at doi:10.1016/ j.jmb.2010.05.057

\section{References}

1. Perutz, M. (1989). Mechanisms of cooperativity and allosteric regulation in proteins. Q. Rev. Biophys. 22, 139-236, doi:10.1017/S0033583500003826.

2. Brunori, M. (1999). Hemoglobin is an honorary enzyme. Trends Biochem. Sci. 24, 158-161, doi: 10.1016/S0968-0004(99)01380-8.

3. Antonini, E. \& Brunori, M. (1971). Hemoglobin and Myoglobin in Their Reactions with Ligands. North Holland, Amsterdam, The Netherlands.

4. Monod, J., Wyman, J. \& Changeux, J. P. (1965). On the nature of allosteric transition: a plausible model. J. Mol. Biol. 12, 88-118, doi:10.1016/S0022-2836(65)80285-6.

5. Perutz, M. F. (1970). Stereochemistry of cooperative effects in haemoglobin. Nature, 228, 726-734, doi: $10.1038 / 228726 a 0$

6. Sawicki, C. \& Gibson, Q. (1976). Quaternary conformational changes in human hemoglobin studied by laser photolysis of carboxyhemoglobin. J. Biol. Chem. 251, 1533-1542.

7. Hofrichter, J., Sommer, J. H., Henry, E. R. \& Eaton, W. A. (1983). Nanosecond absorption spectroscopy of hemoglobin: elementary processes in kinetic cooperativity. Proc. Natl Acad. Sci. USA, 80, 2235-2239, doi:10.1073/pnas.80.8.2235.

8. Murray, L. P., Hofricter, J., Henry, E. R., Ikeida-Saito, M., Kitagishi, K., Yonetani, T. \& Eaton, W. A. (1988). The effect of quaternary structure on the kinetics of conformational changes and nanosecond geminate rebinding of carbon-monoxide to hemoglobin. Proc. Natl Acad. Sci. USA, 85, 2151-2155, doi:10.1073/pnas.85.7.2151.

9. Duddell, D. A., Morris, R. J. \& Richards, J. T. (1979). Ultra-fast recombination in nanosecond laser photolysis of carbonylhemoglobin. J. Chem. Soc., Chem. Commun. 2, 75-76, doi:10.1039/C39790000075.

10. Friedman, J. M. \& Lyons, K. B. (1980). Transient Raman study of the CO-haemoprotein photolysis: origin of the quantum yield. Nature, 284, 570-572, doi:10.1038/284570a0.

11. Friedman, J. M., Scott, T. W., Fisanick, G. J., Simon, S. R., Findsen, E. W., Ondrias, M. R. \& Macdonald, V. W. (1985). Localized control of ligand binding in hemoglobin: effect of tertiary structure on picosecond geminate recombination. Science, 229, 187-190, doi:10.1126/science.4012316.

12. Findsen, E. W., Friedman, J. M., Ondrias, M. R. \& Simon, S. R. (1985). Picosecond time resolved resonance Raman studies of hemoglobin: implications for reactivity. Science, 229, 661-665, doi:10.1126/science.4023704.
13. Jayaraman, V., Rodgers, K. R., Mukerji, I. \& Spiro, T. G. (1995). Hemoglobin allostery: resonance Raman spectroscopy of kinetic intermediates. Science 20, 269, 1843-1848, doi:10.1126/science.7569921.

14. Bjorling, S. C., Goldbeck, R. A., Paquette, S. J., Milder, S. J. \& Kliger, D. S. (1996). Allosteric intermediates in hemoglobin: 1. Nanosecond time-resolved circular dichroism spectroscopy. Biochemistry, 35, 8619-8627, doi:10.1021/bi952247s.

15. Goldbeck, R. A., Esquerra, R. M. \& Kliger, D. S. (2002). Hydrogen bonding to Trp-beta-37 is the first step in a compound pathway for hemoglobin allostery. J. Am. Chem. Soc. 124, 7646-7647, doi:10.1021/ja025855l.

16. Balakrishnan, G., Tsai, C. H., Wu, Q., Case, M. A., Pevsner, A., McLendon, G. L. et al. (2004). Hemoglobin site-mutants reveal dynamical role of interhelical H-bonds in the allosteric pathway: time-resolved UV resonance Raman evidence for intra-dimer coupling. J. Mol. Biol. 340, 857-868, doi:10.1016/j.jmb.2004.05.013.

17. Goldbeck, R. A., Paquette, S. J., Bjorling, S. C. \& Kliger, D. S. (1996). Allosteric intermediates in hemoglobin: 2. Kinetic modeling of $\mathrm{HbCO}$ photolysis. Biochemistry, 35, 8628-8639, doi:10.1021/bi952248k.

18. Svergun, D. I. \& Kock, M. H. J. (2003). Smallangle scattering studies of biological macromolecules in solution. Rep. Prog. Phys. 80, 1735-1782, doi:10.1088/0034-4885/66/10/R05.

19. Svergun, D., Barberato, C. \& Koch, M. H. J. (1995). CRYSOL-a program to evaluate X-ray solution scattering of biological macromolecules from atomic coordinates. J. Appl. Crystallogr. 28, 768-773, doi:10.1107/S0021889895007047.

20. Cammarata, M., Levantino, M., Schotte, F., Anfinrud, P. A., Ewald, F., Choi, J. et al. (2008). Tracking the structural dynamics of proteins in solution using timeresolved wide-angle X-ray scattering. Nat. Methods, 5, 881-886, doi:10.1038/nmeth.1255.

21. Guallar, V., Jarzecki, A. A., Friesner, R. A. \& Spiro, T. G. (2006). Modeling of ligation-induced helix/ loop displacements in myoglobin: toward an understanding of hemoglobin allostery. J. Am. Chem. Soc. 128, 5427-5435, doi:10.1021/ja057318h.

22. Cammarata, M., Lorenc, M., Kim, T. K., Lee, J. H., Kong, Q. Y., Pontecorvo, E. et al. (2006). Impulsive solvent heating probed by picosecond X-ray diffraction. J. Chem. Phys. 124, 124504, doi:10.1063/1.2176617.

23. Jones, C. M., Ansari, A., Henry, E. R., Christoph, G. W., Hofrichter, J. \& Eaton, W. A. (1992). Speed of intersubunit communication in proteins. Biochemistry, 31, 6692-6702, doi:10.1021/bi00144a008.

24. Fader, W. J. (1976). Density perturbations caused by weak absorption of a laser pulse. J. Appl. Phys. 47, 1975-1978, doi:10.1063/1.322922.

25. Henry, E. R. \& Hofrichter, J. (1992). Singular value decomposition: application to analysis of experimental data. Methods Enzymol. 210, 129-192, doi: 10.1016/0076-6879(92)10010-B.

26. Levantino, M., Cupane, A. \& Zimanyi, L. (2003). Quaternary structure dependence of kinetic hole burning and conformational substrates interconversion in hemoglobin. Biochemistry, 42, 4499-4505, doi: 10.1021/bi0272555.

27. Levantino, M., Cupane, A., Zimanyi, L. \& Ormos, P. (2004). Different relaxations in myoglobin after photolysis. Proc. Natl Acad. Sci. USA, 101, 14402-14407, doi:10.1073/pnas.0406062101.

28. Henry, E. R., Jones, C. M., Hofrichter, J. \& Eaton, W. A. (1997). Can a two-state MWC allosteric model explain 
hemoglobin kinetics? Biochemistry, 36, 6511-6528, doi: 10.1021/bi9619177.

29. Henry, E. R., Bettati, S., Hofrichter, J. \& Eaton, W. A (2002). A tertiary two-state allosteric model for hemoglobin. Biophys. Chem. 98, 149-164, doi: 10.1016/S0301-4622(02)00091-1.

30. Makowski, L., Rodi, D. J., Mandava, S., Minh, D. D. L. Gore, D. B. \& Fischetti, R. F. (2008). Molecular crowding inhibits intramolecular breathing motions in proteins. J. Mol. Biol. 375, 529-546, doi:10.1016/j. jmb. 2007.07.075.

31. Esquerra, R. M., Goldbeck, R. A., Reaney, S. H., Batchelder, A. M., Wen, Y. X., Lewis, J. W. \& Kliger, D. S. (2000). Multiple geminate ligand recombinations in human hemoglobin. Biophys. J. 78, 3227-3239, doi:10.1016/S0006-3495(00)76859-7.

32. Kachalova, G. S., Popov, A. N. \& Bartunik, H. D. (1999). A steric mechanism for inhibition of CO binding to heme proteins. Science, 284, 473-476, doi: 10.1126/science.284.5413.473.

33. Balakrishnan, G., Case, M. A., Pevsner, A., Tengroth, X. Z. C., McLendon, G. L. \& Spiro, T. G. (2004). Time-resolved absorption and UV resonance Raman spectra reveal stepwise formation of $t$ quaternary contacts in the allosteric pathway of hemoglobin. J. Mol. Biol. 340, 843-856, doi:10.1016/j. jmb.2004.05.012.

34. Cammarata, M., Eybert, L., Ewald, F., Reichenbach, W., Wulff, M., Anfinrud, P. et al. (2009). Chopper system for time resolved experiments with synchrotron radiation. Rev. Sci. Instrum. 80, 015101, doi: 10.1063/1.3036983.

35. Benesch, R., Macduff, G. \& Benesch, R. (1965). Determination of oxygen equilibria with a versatile new tonometer. Anal. Biochem. 11, 81-87, doi: 10.1016/0003-2697(65)90045-X 